XXXIX "Jaszowiec" International School and Conference on the Physics of Semiconductors, Krynica-Zdrój 2010

\title{
Anharmonic Optical Phonon Effects in ZnO Nanocrystals
}

\author{
S.V. Khusnutdinov ${ }^{a, b, c}$, E. Dynowska ${ }^{b}$, W. ZALeszCZyK ${ }^{b}$, V.P. Makhniy ${ }^{a}$,
} A. WYSMOŁEK ${ }^{c}$ AND K.P. KORONA ${ }^{c, *}$

${ }^{a}$ Yuriy Fedkovych Chernivtsi National University, Kotsubinsky Str., 2, 58012, Chernivtsi, Ukraine

${ }^{b}$ Institute of Physics, Polish Academy of Sciences, al. Lotników 32/46, 02-668 Warszawa, Poland ${ }^{c}$ Institute of Experimental Physics, Faculty of Physics, University of Warsaw, Hoża 69, 00-681 Warszawa, Poland

Zinc oxide $(\mathrm{ZnO})$ is a very promising material for optoelectrical devices operating at the short-wavelength end of the visible spectral range and at the near UV. The Raman scattering studies of ZnO heterolayers formed by isothermal annealing show sharp phonon lines. In addition to the $A_{1}(\mathrm{TO}), E_{1}(\mathrm{TO}), E_{2}^{\mathrm{H}}$, and $E_{1}(\mathrm{LO})$ one-phonon lines, we observed two-phonon lines identified as: $E_{2}^{\mathrm{H}}-E_{2}^{\mathrm{L}}, E_{2}^{\mathrm{H}}+E_{2}^{\mathrm{L}}$, and $2 \mathrm{LO}$ at 332,541 , and $1160 \mathrm{~cm}^{-1}$, respectively (at room temperature). The identification of the $E_{2}^{\mathrm{H}}-E_{2}^{\mathrm{L}}$ peak was confirmed by its thermal dependence. Temperature dependent measurements in the range 6-300 K show that the phonon frequencies decrease with temperature. The $E_{2}^{\mathrm{H}}$ peak is at energy $54.44 \mathrm{meV}\left(439.1 \mathrm{~cm}^{-1}\right)$, at $4 \mathrm{~K}$ and due to phonon-phonon anharmonic interaction, its energy decreases to $54.33 \mathrm{meV}\left(438.2 \mathrm{~cm}^{-1}\right)$ at room temperature. The Grüneisen parameter found for this oscillation mode was $\gamma_{\mathrm{E} 2 \mathrm{H}}=1.1$ at about $300 \mathrm{~K}$. The intensity of the $E_{2}^{\mathrm{H}}-E_{2}^{\mathrm{L}}$ peak increases strongly with temperature and this dependence can be described by the Bose-Einstein statistics with activation energy of $13.8 \mathrm{meV}$ (nearly equal to the energy of the $E_{2}^{\mathrm{L}}$ phonon).

PACS: 73.61.Ga, 74.25.nd, 63.20.kg

\section{Introduction}

The II-VI compounds are well known for their bright luminescence and good photoelectric properties. Between them zinc oxide $(\mathrm{ZnO})$ has special place with its direct and wide band gap (about $3.2 \mathrm{eV}$ at $300 \mathrm{~K}$ ). For example $\mathrm{ZnO}$ diodes are very good detectors and emitters in the short-wavelength end of the visible spectral range and at the near UV [1, 2]. The opto-electronic devices are strongly dependent on electron-phonon scattering thus the lattice dynamics of the material is very important.

The Raman scattering spectroscopy is one of the most popular phonon research techniques. The temperature dependences of the phonon frequency follow from anharmonic terms in the vibrational Hamiltonian of the crystal lattice. The anharmonicity gives rise to the thermal expansion of the lattice and to the phonon-phonon interaction.

In the wurtzite (hexagonal) phase, the $\mathrm{ZnO}$ primitive cell consists of two $\mathrm{Zn}-\mathrm{O}$ pairs (twice more than in the case of the cubic phase) so nine optical modes appear. The symmetry representations are: $\Gamma_{\text {opt }}=A_{1}+E_{1}+$ $2 B_{1}+2 E_{2}$. The $B_{1}$ modes are optically inactive. The $E_{2}$ modes are nonpolar, but they give strong signal in the Raman spectra (especially the high-frequency $E_{2}^{\mathrm{H}}$ mode). The polar $A_{1}$ and $E_{1}$ modes can be longitudinal (LO), and transverse (TO) [3].

\section{Samples}

Substrates measuring $4 \times 4 \times 1 \mathrm{~mm}$ were cut from bulk crystals of cubic ZnSe, grown by the Bridgman method

\footnotetext{
* corresponding author; e-mail: kkorona@fuw.edu.pl
}

from stoichiometric melts. The substrate surface was mirror-smooth, and the substrates showed bulk photoluminescence (PL) under excitation by the $337 \mathrm{~nm}$ nitrogen laser line. Zinc oxide layers were produced by isovalent substitution method [4]. Isothermally annealing was realized in air at temperatures from 850 to $1050^{\circ} \mathrm{C}$, and time in the range of 5-30 min. The width of the $\mathrm{ZnO}$ layers was from 0.1 to $1 \mu \mathrm{m}$ depending on growth conditions.

X-ray diffraction (XRD) characterization was conducted in X'Pert MPD Philips Analytical Rentgen diffractometer in $\theta-2 \theta$ configuration. The lattice parameters were determined by optimization to powder-pattern indexing method [5].

The XRD of the $\mathrm{ZnO}$ heterolayers (Fig. 1) revealed that the $\mathrm{ZnO}$ is polycrystalline. This conclusion is based

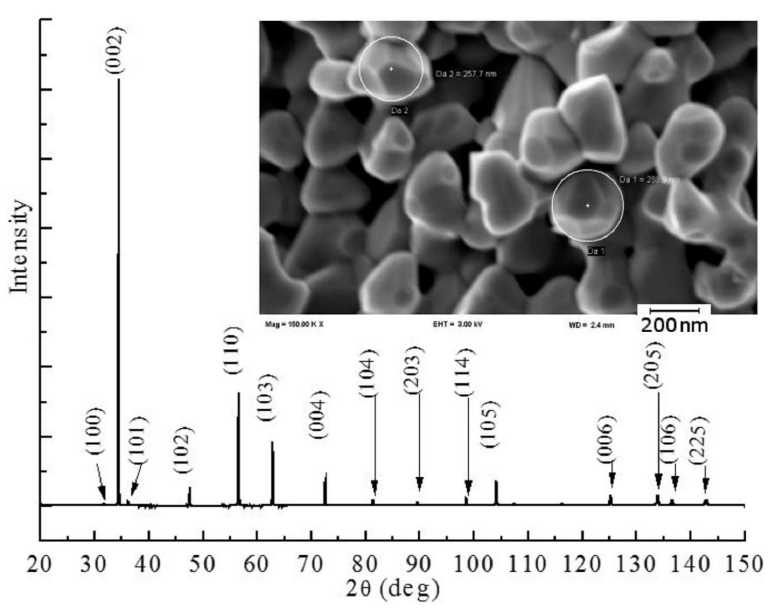

Fig. 1. X-ray diffraction spectrum of the $\mathrm{ZnO}$ heterolayers formed on $\mathrm{ZnSe}$. The inset: electron microscope picture of the sample. 
on the presence of many reflections from different crystallographic planes. All diffraction peaks were attributed to the $\mathrm{ZnO}$ in wurtzite structure. The strongest peak comes from (002) plane reflection which suggests that the most of the crystallites have grown along the $c$ axis.

The lattice parameters were determined: $a=3.2506 \pm$ $0.0002 \AA$ and $c=5.2063 \pm 0.0003 \AA$. It means that the $a$ constant of the sample is slightly higher than the standard value $(3.2498 \AA)$ and the $c$ constant is nearly equal (within the experimental error) to the standard value

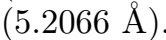

Morphology of the $\mathrm{ZnO}$ samples was examined by scanning electron microscope Carl Zeiss Neon 40. The SEM measurements confirmed that the $\mathrm{ZnO}$ layers are polycrystalline. The average size of the nanocrystals that form the layer is of the order of 200-300 $\mathrm{nm}$ (see inset in Fig. 1).

\section{Measurements and discussion}

The Raman spectroscopy was measured in backscattering geometry with use of a TRIAX 550 spectrometer with a $\mathrm{LN}_{2}$-cooled charge-coupled device (CCD) detector. For an exact calibration of the wavelength, mercury spectral lines were recorded. A $532 \mathrm{~nm}$ light of a frequency doubled Nd:YAG laser was used. Measurements were made in a continouos-flow helium cryostat (Konti Mikro) in 6-300 $\mathrm{K}$ temperature range.

Due to the low strain, the phonon modes were sharp and well visible in our samples. Few peaks related to $E_{1}$, $E_{2}$ and $A_{1}$ phonon modes were observed. The strongest peak at $438.2(5) \mathrm{cm}^{-1}($ at $300 \mathrm{~K})$ was the $E_{2}^{\mathrm{H}}$ mode. It had width of $9 \mathrm{~cm}^{-1}$ (FWHM). The $E_{1}$ (TO), $A_{1}$ (TO) and $E_{1}(\mathrm{LO})$ peaks were visible at $381 \mathrm{~cm}^{-1}, 410 \mathrm{~cm}^{-1}$, and $585 \mathrm{~cm}^{-1}$, respectively. The peak positions were in agreement with previously published data [6-8]. The Raman spectra measured at different temperatures are plotted in Fig. 2.

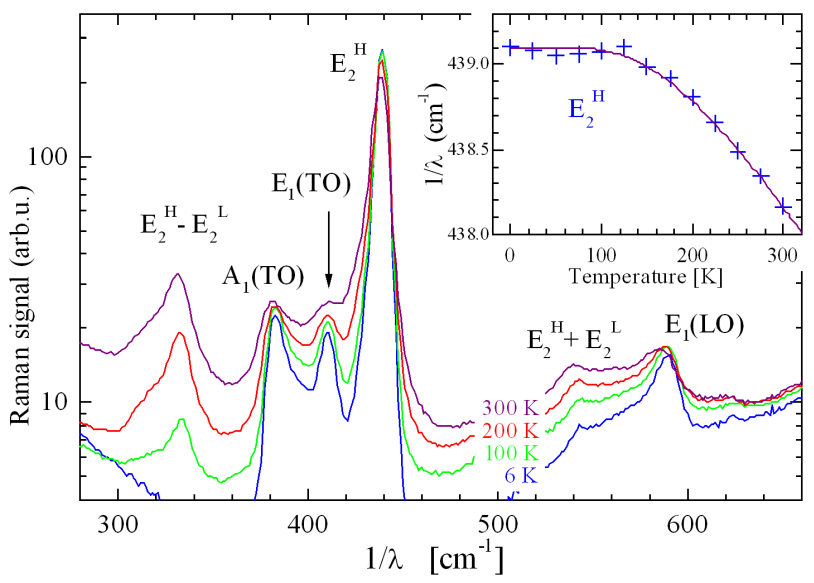

Fig. 2. Raman spectra at different temperatures. The inset: thermal dependence of the $E_{2}^{\mathrm{H}}$ peak energy: crosses - data points, line - fit of Eq. (1).
During heating, we observed red-shifts of the phonon peaks (in agreement with previous reports $[9,10]$ ), increase of widths and changes of heights of the phonon peaks (see Figs. 2 and 3 ). At $4 \mathrm{~K}$, the $E_{2}^{\mathrm{H}}$ peak was at $1 / \lambda=439.1(5) \mathrm{cm}^{-1}$ (energy $=54.44(5) \mathrm{meV}$ ). At the room temperature, the $E_{2}^{\mathrm{H}}$ energy decreased and the difference was $0.9(1) \mathrm{cm}^{-1}$. The temperature-related frequency shifts between $6 \mathrm{~K}$ and $300 \mathrm{~K}$ were: $1.6 \mathrm{~cm}^{-1}$, $0.5 \mathrm{~cm}^{-1}$, and $4 \mathrm{~cm}^{-1}$, for $A_{1}(\mathrm{TO}), E_{1}(\mathrm{TO})$, and $\mathrm{LO}$, respectively.

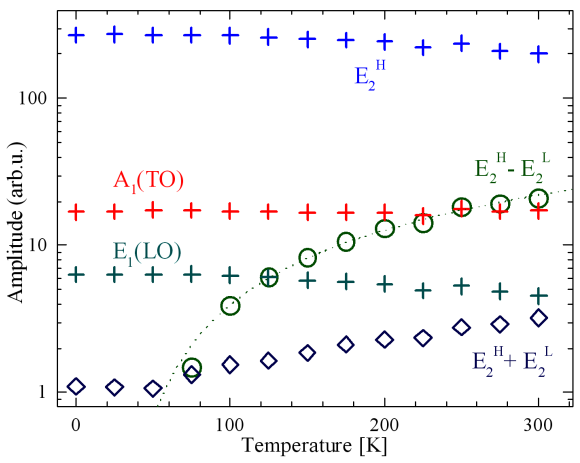

Fig. 3. Dependence of the Raman peak amplitudes versus temperature. Dotted line is fit of Eq. (2).

The thermal frequency change coefficients $\phi=$ $d \ln (\omega) / \mathrm{d} T$ near the room temperature were calculated: $2.8(2), 0.2(2), 1.5(1), 4.5(5)\left[\times 10^{-5} \mathrm{~K}^{-1}\right]$ for $A_{1}(\mathrm{TO})$, $E_{1}(\mathrm{TO}), E_{2}^{\mathrm{H}}$, and LO modes, respectively. Comparing coefficient $\phi$ with volume thermal expansion coefficient $\beta=1.4 \times 10^{-5} \mathrm{~K}^{-1}[11]$, one can obtain the Grüneisen parameter: $\gamma=\phi / \beta$. These parameters were $2,0.1,1.1$, and 3.2 for $A_{1}(\mathrm{TO}), E_{1}(\mathrm{TO}), E_{2}^{\mathrm{H}}$, and LO, respectively. The high $\gamma$ value for the LO peak was probably an artifact caused by existence of two LO modes with slightly different energies. During increase of temperature, the amplitude of the low-energy $A_{1}(\mathrm{LO})$ mode grew in respect of high-energy $E_{1}(\mathrm{LO})$ mode. Such $A_{1}(\mathrm{LO})$ behavior was already observed in above room temperature measurements [10].

The temperature dependences of the phonon frequency and linewidth come from anharmonic terms in the vibrational Hamiltonian of the crystal lattice $[12,13]$ leading to phonon-phonon interactions. These processes could explain both energy and width dependences of the Raman lines. The thermal dependences should be proportional to the numbers of phonons, as given by

$$
E(T)=E_{0}-\frac{b}{\exp (\theta / T)-1},
$$

where the oscillator energy, $E_{0}$, thermal change, $b$, and phonon activation temperature, $\theta$, were the fitting parameters. The obtained activation energy, $k_{\mathrm{B}} \theta=$ $64(5) \mathrm{meV}$, is slightly higher than $E_{2}^{\mathrm{H}}$ energy $(54 \mathrm{meV})$ but close to $E_{2}^{\mathrm{H}}+E_{2}^{\mathrm{L}}$ energy $(67 \mathrm{meV})$.

In addition to one-phonon peaks, it was possible to observe few two-phonon peaks: at $332 \mathrm{~cm}^{-1}, 541 \mathrm{~cm}^{-1}$, and 
$1160 \mathrm{~cm}^{-1}$. The phonon-phonon interactions were due to anharmonic terms in the potential. The peaks were previously reported [14] and attributed to the second-order features. The $332 \mathrm{~cm}^{-1}$ peak was attributed to two-phonon scattering from $\Sigma-M$ branch having maximum in phonon density of states at $160 \mathrm{~cm}^{-1}$ [14]. The $541 \mathrm{~cm}^{-1}$ peak was attributed to two-phonon scattering on $270 \mathrm{~cm}^{-1}$ phonons near the $M$ point [14]. The $1160 \mathrm{~cm}^{-1}$ peak was attributed to two-phonon scattering on LO phonons from $\Gamma$ point. We agree with the last identification but we suggest that $332 \mathrm{~cm}^{-1}$ and $541 \mathrm{~cm}^{-1}$ were anti-Stokes and Stokes lines of photon scattering on two $\left(E_{2}^{\mathrm{H}}\right.$ and $\left.E_{2}^{\mathrm{L}}\right)$ phonons. It means that the $332 \mathrm{~cm}^{-1}$ line was due to absorption of $E_{2}^{\mathrm{L}}$ phonon with simultaneous emission of $E_{2}^{\mathrm{H}}\left(E_{2}^{\mathrm{H}}-E_{2}^{\mathrm{L}}\right.$ process $)$. The difference of $E_{2}^{\mathrm{H}}$ energy $438 \mathrm{~cm}^{-1}$ and $E_{2}^{\mathrm{L}} 102 \mathrm{~cm}^{-1}$ gives value close to the $332 \mathrm{~cm}^{-1}$. The $541 \mathrm{~cm}^{-1}$ line is due to simultaneous emission of $E_{2}^{\mathrm{L}}$ and $E_{2}^{\mathrm{H}}$ phonons $\left(E_{2}^{\mathrm{H}}+E_{2}^{\mathrm{L}}\right.$ process $)$. The $1160 \mathrm{~cm}^{-1}$ line is due to simultaneous emission of the two LO phonons (about $580 \mathrm{~cm}^{-1}$ each).

Moreover our thesis about the $E_{2}^{\mathrm{H}}-E_{2}^{\mathrm{L}}$ line origin is strongly supported by temperature dependence of scattering probability (see Fig. 3).

The data points in Fig. 3 were obtained by fitting procedure. Background and a set of spectral curves were fitted simultaneously to the spectra measured at different temperatures.

It is visible that the amplitude of the $E_{2}^{\mathrm{H}}-E_{2}^{\mathrm{L}}$ peak strongly depended on temperature. It increased an order of magnitude between $80 \mathrm{~K}$ and $300 \mathrm{~K}$, as expected for anti-Stokes processes. This observation is supported by other reports $[9,10]$. For example, at $873 \mathrm{~K}$ the $330 \mathrm{~cm}^{-1}$ peak was observed to be of the same height as the $E_{2}^{\mathrm{H}}$ peak $[10]$.

The height of the $E_{2}^{\mathrm{H}}-E_{2}^{\mathrm{L}}$ peak should depend on the number of the $E_{2}^{\mathrm{L}}$ phonons. This number, $n_{s}$, is given by the Bose-Einstein statistic

$$
n_{\mathrm{S}}(\Omega, T)=\frac{1}{\exp \left(\hbar \Omega / k_{\mathrm{B}} T\right)-1} .
$$

The curve (2) was fitted to the experimental dependence (see dotted line at Fig. 3.). The energy obtained from the fit, $\hbar \Omega=13.8 \pm 2 \mathrm{meV}\left(1 / \lambda=111 \pm 15 \mathrm{~cm}^{-1}\right)$ was nearly equal to the expected energy of the $E_{2}^{L}$ phonons $\left(98 \mathrm{~cm}^{-1}[7]\right.$ to $\left.102 \mathrm{~cm}^{-1}[8]\right)$.

\section{Conclusion}

Several one- and two-phonon lines in nanocrystalline $\mathrm{ZnO}$ were observed by the Raman spectroscopy and identified. Temperature dependence analyzed for the Raman peaks showed that the energy of the peaks decreased significantly with increase of temperature. The obtained Grüneisen parameters were 2, 0.1, 1.1, and 3.2 for $A_{1}(\mathrm{TO}), E_{1}(\mathrm{TO}), E_{2}^{\mathrm{H}}$, and LO modes, respectively.

Two-phonon processes were observed: absorption of the $E_{2}^{\mathrm{L}}$ phonon with simultaneous emission of the $E_{2}^{\mathrm{H}}$ phonon, at $332 \mathrm{~cm}^{-1}$, simultaneous emission of the $E_{2}^{\mathrm{L}}$ and $E_{2}^{\mathrm{H}}$ phonons, at $541 \mathrm{~cm}^{-1}$, and emission of two LO phonons, at $1160 \mathrm{~cm}^{-1}$. The amplitudes of the Stokes peaks were weakly sensitive to temperature, whereas amplitude of the Stokes + anti-Stokes peak $E_{2}^{\mathrm{H}}-E_{2}^{\mathrm{L}}$ increase strongly during heating.

Both the thermal frequency change and two-phonon processes arise from the phonon-phonon interactions which are due to anharmonic terms in the potential.

\section{Acknowledgments}

S.V.K. work was supported by the International Visegrad Fund. K.P.K. work was supported by EU project No. MTKD-CT-2005-029671.

\section{References}

[1] H. Morkoç, Ü. Özgür, Zinc Oxide Fundamentals. Materials and Device Technology, Wiley-VCH, Weinheim 2009.

[2] Ü. Özgür, Ya.I. Alivov, C. Liu, A. Teke, M.A. Reshchikov, S. Dođan, V. Avrutin, S.-J. Cho, H. Morkoç, J. Appl. Phys. 98, 041301 (2005).

[3] T.C. Damen, S.P.S. Porto, B. Tell, Phys. Rev. 142, p. 570 (1966)

[4] V.P. Makhnii, M.M. Sletov, S.V. Khusnutdinov, Inorg. Mater. 43, 1304 (2007).

[5] W. Paszkowicz, J. Appl. Crystallogr. 20, 166 (1987).

[6] V.A. Nikitenko, V.G. Plekhanov, S.V. Mukhin, M.V. Tkachev, J. Appl. Spectrosc. 63, 290 (1996).

[7] B.H. Bairamov, A. Heinrich, G. Irmer, V.V. Toporov, E. Ziegler, Phys. Status Solidi B 119, 227 (1983).

[8] N. Ashkenov, B.N. Mbenkum, C. Bundesmann, V. Riede, M. Lorenz, D. Spemann, E.M. Kaidashev, A. Kasic, M. Schubert, M. Grundmann, G. Wagner, H. Neumann, V. Darakchieva, H. Arwin, B. Monemar, J. Appl. Phys. 93, 126 (2003).

[9] W. Gebicki, K. Osuch, C. Jastrzebski, Z. Golacki, M. Godlewski, Superlattices Microstruct. 38, 428 (2005).

[10] Jianfeng Xu, W. Ji, X.B. Wang, H. Shu, Z.X. Shen, S.H. Tang, J. Raman Spectrosc. 29, 613 (1998).

[11] R.R. Reeber, J. Appl. Phys. 41, 5063 (1970)g.

[12] J. Menéndez, M. Cardona, Phys. Rev. B 29, 2051 (1984).

[13] M. Balkanski, R.F. Wallis, E. Haro, Phys. Rev. B 28, 1928 (1983)

[14] J.M. Calleja, M. Cardona, Phys. Rev. B 16, 3753 (1977). 